\title{
Oriented cell divisions in epithelia: from force generation to force anisotropy by tension, shape and vertices
}

van Leen Eric Victor ${ }^{1,2}$, di Pietro Florencia ${ }^{1,2}$, Bellaïche Yohanns ${ }^{1,2,3}$

1 : Institut Curie, PSL Research University, CNRS UMR 3215, INSERM U934, F-75248 Paris Cedex 05, France.

2 : Sorbonne Universités, UPMC Univ Paris 06, CNRS UMR 3215, INSERM U934, F-75005, France.

3: Corresponding author: yohanns.bellaiche@curie.fr

\begin{abstract}
Mitotic spindle orientation has been linked to asymmetric cell divisions, tissue morphogenesis and homeostasis. The canonical pathway to orient the mitotic spindle is composed of the cortical recruitment factor NuMA and the molecular motor dynein, which exerts pulling forces on astral microtubules to orient the spindle. Recent work has defined a novel role for NuMA as a direct contributor to force generation. In addition, the exploration of geometrical and physical cues combined with the study of classical polarity pathways has led to deeper insights into the upstream regulation of spindle orientation. Here, we focus on how cell shape, junctions and mechanical tension act to orient spindle pulling forces in epithelia, and discuss different roles for spindle orientation in epithelia.
\end{abstract}

\section{Introduction}

During animal development, complex epithelial structures are formed by regulating the spatiotemporal dynamics of cell rearrangements, cell shape changes, apoptosis and cell division [1-4]. Intensive work in the last 20 years highlighted that the orientation of cell division can play essential roles in asymmetric cell fate divisions and tissue architecture, and allowed the establishment of a paradigm for spindle orientation within the cell $[3,5,6]$. The axis of division is set up by cortical pulling forces acting on astral microtubules (MT) $[7,8]$. Therefore, the orientation of division can be ensured by locating pulling force generators at specific cortical locations within the cell. While force generation has been attributed to the MT minusend directed motor dynein, the recruitment task was assigned to NuMA (Mud in Drosophila, and Lin-5 in C. elegans), which in turn can be recruited to the cortex by LGN (Pins in Drosophila, and GPR-1/2 in C. elegans) and the heterotrimeric G protein Gai [3]. Recently, the relative roles of NuMA and dynein in force generation for spindle orientation have been further deciphered.

In symmetric cell divisions in epithelia, mitotic spindles are stereotypically oriented with respect to polarity axes within the tissue $[9,10]$. Classical observations of dividing single cells have led to the proposition of Hertwig's rule, which states that cells divide according to their longest axis $[11,12]$. While true in many contexts, cell divisions that challenge this rule have been reported $[9,13,14]$. Recent work has provided novel insights into whether and how epithelial cells adhere to Hertwig's rule, as well as how tension and polarity are integrated by cells to orient their spindles. 
In this review, we first discuss how the cortical forces necessary for oriented cell divisions (OCD) are generated within the cell. Second, we describe recent data exploring how cell shape and local or global tissue tension influence spindle orientation in symmetric divisions in epithelia. Finally, we describe the function of spindle orientation in epithelial morphogenesis and homeostasis. For a review of mitotic spindle orientation in asymmetric cell divisions as well as a more complete oversight of the proteins involved in spindle orientation, we refer the readers to recent reviews $[3,6]$.

\section{How are spindle orientation forces generated within the cell?}

Mitotic spindle orientation commonly requires the exertion of cortical forces on the astral MT $[7,8]$. The current paradigm for force generation involves the recruitment of dynein and its cofactor dynactin to the cell cortex $[7,8,15-18]$. However, recent studies have indicated that cortical recruitment of dynein is insufficient for OCD [19,20]. Here we discuss recent findings on dynein activity and how its interactors assist dynein in force generation [17,19-22].

\section{Dynein force regulation and spindle orientation}

Work in C. elegans and mammalian cells has led to the identification of different dynein regulators required for spindle orientation such as LIS-1, Nde1/NdeL1, and a subset of dynactin subunits including p150 [23-27]. Recent structural analyses showed that dynein can form a ternary complex with dynactin and a cargo adaptor [28]. In vitro reconstitution indicates that this ternary complex is highly processive and capable of transporting cargo over large distances (Figure 1A) [29]. Two recent structures of dynein/dynactin complexed with the cargo adapters HOOK3 and BICD2 show that their interaction is facilitated by the coiled-coils of the adapter proteins which run along the length of dynactin [28]. As BICD2 is not essential for spindle orientation in cultured cells, this raises the question whether HOOK3 or another cargo adapter activates dynein during spindle orientation [27,30]. Furthermore, depending on the cargo adapter, one or more dyneins can be part of the ternary complex. Complexes with two dyneins generate higher forces and walk faster on microtubules [28]. The binding of a second dynein is facilitated by the CAPZ $\beta$ subunit of the dynactin barbed end (Figure 1A) [28]. Interestingly, recent experiments have revealed that CAPZ $\beta$ knock-down leads to spindle misorientation in vivo, suggesting that the capacity of binding two dyneins contributes to division orientation regulation [27].

\section{New roles for NuMA during spindle orientation}

The respective roles of dynein and NuMA during spindle orientation have recently been investigated by using optogenetics to position components of the spindle orientation machinery at the cortex. In these studies, the authors independently found that optogenetic recruitment of NuMA to the cortex was sufficient to orient the mitotic spindle [17,22]. By contrast and in agreement with other studies [19,20], recruitment of the dynein heavy chain/dynactin to the cortex was insufficient to generate pulling forces on the mitotic spindle $[17,22]$. This suggests that NuMA is required not only for dynein recruitment, but also for force production without additional upstream components.

\section{NuMA coiled-coil and MT binding domains are essential for spindle orientation}

All NuMA homologs have a coiled-coil domain which has been shown to be required for spindle orientation in Drosophila and HeLa cells [7,17,31]. Therefore, in the light of the recently established ternary dynein complex structure described above, NuMA is a potential candidate to work as a regulator of dynein activity (Figure 1B). Furthermore, Seldin and colleagues showed that NuMA localizes to MT tips in interphase and pro-metaphase cells, and that 
deletion of the domain responsible for this localization (AA:1811-1985 in human) impaired spindle orientation in cultured keratinocytes and in vivo [20]. In addition, Gallini and colleagues found a second NuMA MT binding domain, (AA: 2002-2115 in human) which is also essential for spindle orientation in cultured cells [19,21]. Accordingly, this second MT binding is necessary to orient the spindle upon optogenetic recruitment of NuMa to the cortex [17]. Exactly how NuMA and its MT binding domains assists in spindle orientation remains to be better defined. For instance, MT tip localized NuMA could regulate MT growth dynamics, cortical capture or cortical dwell time of astral MTs which have all been proposed to regulate spindle orientation $[3,32,33]$.

In summary, these recent data illustrate that MT pulling force generation does not solely rely on dynein and its interaction with p150 and LIS1, but also requires NuMA. Direct analyses of astral MT dynamics and measurement of astral MT associated forces, combined with the depletion of the aforementioned NuMA domains and optogenetic recruitment will greatly contribute to a better understanding of dynein regulation in spindle orientation.

\section{How do cell shape and tissue tension result in anisotropic spindle orientation forces?}

The first exploration of the influence of cell geometry on division orientation was performed at the end of the $19^{\text {th }}$ century. In these experiments, rounded amphibian eggs were flattened between two glass slides resulting in the cleavage planes of the first two divisions being reproducibly positioned perpendicular to the long axis of the egg [11,34,35]. Predicting the orientation of division based on the cells' long axis later became known as Hertwig's rule. In depth study of the underlying mechanism was possible after advances in microfabrication enabled standardized regulation of cell shape and the application of defined mechanical stress. Combined with automated measurements of cell division orientation and physical modeling this led to important advances in linking geometry and division orientation $[12,36-$ 38].

Théry and colleagues used fibronectin adhesive micropatterns to standardize the 2D shape of single adhesive cells to study the influence of 2D cell shape on spindle orientation. The authors found that it is not cell shape, but the specific distribution of actin rich retraction fibers connecting the mitotic cell to the substrate, which best predicts the angles of spindle orientation $[37,38]$. As the distribution of retraction fibers is controlled by the shape of the adhesion pattern, these fibers were proposed to provide a memory of the interphase adhesion pattern of the cell [36-38]. Retraction fibers direct the distribution of cytoplasmic actin clouds that bias the forces exerted on astral MT. In particular, the unconventional MT bound Myosin10 localizes to these actin clouds where it modulates astral MT dynamics [32,36]. This mechanism was proposed to contribute to spindle orientation in parallel to the classical LGN/NuMA/dynein pathway [32,39]. Following Hertwig's seminal experiments, Minc and colleagues squeezed sea urchin eggs into microfabricated wells resulting in division orientation according to the long axis created by the applied external constraints [12]. By using quantitative modeling, they proposed that the applied rotational forces on centrosomes scale with MT length, linking cell geometry with division orientation for each specific cell shape in single cells [12].

In epithelia, several studies initially identified major roles for planar cell polarity (PCP) and apical-basal $(A B)$ polarity in orienting cell divisions $[9,10]$. These studies were instrumental in analyzing spindle orientation in multicellular contexts as well as the mechanisms of spindle 
orientation with respect to the apical-basal axis. Recent work explored whether and how geometry and tension play roles in regulating spindle orientation in epithelial tissues like in single cells, which we will discuss in the next sections.

\section{Two axes of spindle orientation in epithelia: apical-basal and planar}

In epithelia, mitotic spindles are typically oriented perpendicular to the apical-basal axis, which is critical to maintain both daughter cells in the plane of the epithelium. This orientation is generally dependent on the lateral distribution of pulling forces [40-42]. Interestingly, when the actomyosin machinery necessary for cell rounding is impaired, the geometry can override the lateral pulling forces $[40,41,43]$. Recently, Box and colleagues found that $A B$ division orientation can respond to changes in cell geometry in mouse basal epidermal progenitors. Mutants for the PCP protein Vangl2 fail to close their neural tube, which in turn alters cell geometry, thereby indirectly perturbing division orientation relative to the $A B$ axis [44].

Spindle orientation is also regulated in the plane of the tissue (planar orientation, Figure 2 top center). In most tissues, planar cell divisions follow Hertwig's rule aligning the mitotic spindle with the cell interphase long axis (Figure 2A, 3A) [45-47]. A large variety of cell anisotropies exist in tissues, ranging from highly anisotropic (elongated) cells to almost isotropic (round) cells [7,48-50]. Regardless of their interphase shape, cells round up during mitosis by increasing osmotic pressure and stiffening their actomyosin cortex $[51,52]$. Rounding during mitosis poses the question of how the interphase long axis is sensed in cells that orient the spindle in the planar axis according to the long axis rule [40,51,53]. Furthermore, epithelial cells are subjected to mechanical forces, which can directly affect planar spindle orientation and modulate cell shapes (Figure 3B) [4]. Central questions in the field are therefore whether tissue forces are able to orient the spindle, and whether this effect is dependent on cell shape changes.

In single adhesive cells, applying external forces without affecting cell shape was able to induce spindle rotation independently of cell shape [36]. Initial studies in the context of Zebrafish epiboly, and in cultured epithelial monolayers stretched $30 \%$, indicated that tension is able to orient cell divisions, while also orienting cell shape $[54,55]$. In the next section, we discuss several recent studies that investigated how epithelial cells sense their interphase cell shape, as well as how cell divisions are oriented by cell shape and/or tension $[7,13,14,48,50,54]$.

\section{Sensing interphase cell shape in mitosis}

Work in the Drosophila notum epithelium uncovered a mechanism that translates the interphase long axis into localized forces which can orient the mitotic spindle. This mechanism is based on the localization of Mud/NuMA to tricellular junctions (TCJ, the junctions where three cells come together). This localizes the forces exerted on astral MT to TCJ, leading to spindle orientation according to TCJ distribution, which generally aligns with the cell long axis (Figure 2B). In cases where the angle predicted by cell shape and the distribution of TCJ differ by more than 45 degrees, cells divide according to the TCJ distribution showing that the orientation of TCJ is a better predictor for spindle orientation than cell shape [7]. This new read-out of cell shape has later become known as the "TCJ rule".

\section{Tension vs shape in spindle orientation}

To analyze whether tension can orient the spindle independently of cell shape, a recent study subjected the Xenopus embryo animal cap to an uniaxial stretch $(20 \%)$ with a PDMS based device [50]. The externally applied stretch induced both cell shape elongation and cell division 
orientation along the axis of stretch. By comparing cell shape, cell area and the position of the TCJ vertices, the investigators found that TCJ align with the principal axis of local stress and are a better predictor of spindle orientation than global tissue stress $[50,56]$. Indeed, in cells that do not elongate along the axis of stretch, the spindle is not oriented in the axis of stretch, but according to TCJ distribution (Figure 2B, 3C). Mechanistically, TCJ are enriched in Cadherin and LGN, and this distribution is necessary to control planar spindle orientation in this context [50]. In contrast to the findings in Xenopus epithelia, dividing cells within MDCK monolayers subjected to low uniaxial stretch (15\%), oriented their spindle with respect to the stretch axis, independently of cell shape. The mechanism proposed here involves both Cadherin and the polarization of LGN towards the bicellular junctions (BCJ) that are perpendicular to the axis of stretch (Figure 3D) [48]. Therefore, the same molecules mediate the link between the orienting cue (geometry or tension) and force generation by localizing to TCJ or BCJ in Xenopus and MDCK cells respectively.

The link between tension and planar spindle orientation has been also studied in developing tissues which undergo tissue elongation or experience localized tensile stress. In the case of the Drosophila embryo, cells that are adjacent to high tensile supracellular actomyosin cables divide according to their long shape axis only if they are highly elongated. By contrast, slightly elongated cells divide according to the tension cue provided by the actomyosin cables, which run perpendicular to cell shape (Figure 2C) [13]. Another tension dependent mechanism orienting cell divisions was identified in the elongating Drosophila follicular epithelium (FE). In these cells, the cell long axis is perpendicular to the axis of tissue elongation and thus to the stretch force [14]. The embryo and FE constitute immature epithelia which do not harbor classical BCJ and TCJ septate junctions. No clear crescents or TCJ accumulation of LGN or NuMA could be observed and these proteins were not required for planar spindle orientation. In mature epithelia which do have septate junctions (larval wing disc, pupal wing and notum tissues), Mud is enriched at the level of the tricellular septate junction. Therefore, different mechanisms might orient cell divisions according to tension in immature as compared to mature epithelial tissues. In the embryo, the actomyosin cable is proposed to orient the spindle by capturing one centrosome in an actin network, while in the FE, anisotropic cortical stiffness has been proposed to explain the bias in spindle orientation $[13,14]$.

What emerges from these experiments is that no clear one-size fits all rule can be applied. In highly elongated cells, the predominant cue seems to be cell shape, independently of the mechanism used by cells to interpret this cue $[7,13,50]$. In cells with moderate anisotropy, different scenarios are possible: i) in immature epithelial tissues or MDCK layers, where spindle orientation regulators do not localize to TCJ, spindle orientation responds to global tension independently of cell shape; ii) in mature epithelia, cells use TCJ bipolarity, which aligns with local tension, as the main cue for spindle orientation (Figure 2, 3) [7,13,50]. In this view, one could speculate that TCJ hot spots of LGN or NuMA are more efficient to orient the spindle than other mechanisms that depend on actomyosin forces. Actomyosin based spindle orientation mechanisms remain to be defined in epithelia: could actin clouds and Myosin-10 be involved in spindle orientation as observed in cultured cells?

\section{Function of mitotic spindle orientation in epithelia}

A central question in the field of mitotic spindle orientation is the function of OCD in epithelial tissues. Multiple roles have been proposed such as tissue layering, dissipation of tissue stress, changes in tissue morphology, and homeostasis (Figure 4) [4,41,47,54,57]. 
OCD were proposed to be required for axis elongation during Zebrafish gastrulation [9]. The PCP pathway can regulate spindle orientation through Mud and via the Anthrax2 receptor $[58,59]$. Knock down of the PCP protein Dishevelled resulted in a shortened anterior-posterior axis [9]. Yet, more directly perturbing spindle orientation by impairing dynein or NuMA function did not affect body axis elongation, challenging the idea that spindle orientation is involved in tissue elongation during Zebrafish gastrulation $[58,60]$. In Drosophila wing imaginal disc, cells in the central wing pouch divide preferentially along the proximal-distal axis, while cells in the periphery divide tangentially along the local tissue boundary $[10,46,49]$. In this tissue, spindle orientation impairment due to loss of Mud/NuMA leads to the formation of smaller but normally shaped wings without gross phenotypic defects (Figure 4D-G) [61]. Spindle misorientation can result in basally born daughter cells being lost from the tissue possibly explaining the observed size reduction $[41,61]$. As global tissue stress regulates OCD [10,46,49], the authors proposed that loss of OCD contributing to tissue elongation can be buffered by an increase in tissue stress and cell rearrangements contributing to tissue elongation [61]. Therefore, planar division within the wing disc epithelium might serve to aid planar tissue extension and reduce cell loss.

In the developing Zebrafish embryonic surface layer (pre-EVL), Xiong and colleagues proposed that OCD contribute to the layering of the tissue. In this model system, the cell shape predicts the orientation of division with squamous and cuboidal cells dividing in perpendicular directions (Figure 4A) [5]. Cell divisions in cuboidal cells contributed to layering as one of the two daughter cells was displaced from the outer layer into a new layer below. Furthermore, cell division orientation was proposed to buffer changes in the global geometry of the embryo, permitting successful epiboly [4].

In the Zebrafish EVL, cell divisions tend to orient along the axis of tension during epiboly (Figure 4B) [54] and in stretched MDCK monolayers, cell divisions reorient according to the axis of stretch (Figure 3D, 4C) [42,55]. These orientations have been proposed to relieve tissue stress by increasing the number of cells along the stress axis $[54,55]$.

Fine tuning of $A B$ spindle orientation is essential to ensure faithful epithelial structure. During mouse embryonic epithelial development, a switch in spindle orientation, from parallel to perpendicular to the epithelial plane, is necessary for progenitor cells differentiation and skin stratification [62]. Impaired $A B$ spindle orientation can also lead to daughter cells being aberrantly displaced out of the epithelial layer [41]. Therefore, OCD have also been proposed to prevent tumor initiation and/or tumor spreading. Nakajima and colleagues showed that by perturbing spindle orientation and inhibiting apoptosis, tumor like masses can form in fly wing disc epithelia (Figure 4G) [41]. Alternatively, tumor spreading can also be prevented by reintegration of cells after misoriented divisions. In the Drosophila FE, the embryonic ectoderm, the neuroepithelium and the wing disc, misplaced daughter cells can reintegrate in the tissue (Figure 4G) [61,63]. Further exploration of cell reintegration upon division misorientation will help to understand how epithelial tissues can prevent out of plane born daughter cells from becoming tumorigenic. Finally, how epithelial homeostasis and morphogenesis are affected by aberrant cell loss due to $A B$ spindle misorientation remains to be further investigated.

\section{Future directions}

In summary, considerable progress has been made in understanding the mechanisms and roles of mitotic spindle orientation during symmetric epithelial cell division. Accordingly, 
complementary in vitro and in vivo studies have put forward novel hypotheses. For example, cortical clustering of NuMA is proposed to be critical for spindle orientation in cultured cells, while in epithelial tissues TCJ localization of spindle guidance cues seems to be a predominant mechanism to orient the spindle $[17,19]$. This suggests that NuMA clustering at TCJ, rather than homogenous cortical localization, could be more efficient to generate pulling forces in epithelia. Better characterization of the upstream regulators of spindle orientation and of the mechanisms of force generation will extend our understanding of the many roles of spindle orientation in multicellular contexts. Another exciting direction will be to explore how the interplay between tissue mechanics, vertex dynamics and apoptosis influences spindle orientation.

\section{Acknowledgements}

We thank Floris Bosveld for critical reading of the manuscript. Work in Y.B lab is supported by ERC Advanced (TiMorph, 340784), ARC (PGA1 RF20180206793), ANR Labex DEEP 11LBX-0044, ANR-10-IDEX-0001-02 PSL grants for funding. E.v.L. is supported by the Marie Skłodowska-Curie Actions Innovative Training Network 675407 and F.d.P. by the Fondation pour la Recherche Medicale (FRM) postdoctoral grant SPF20170938661. 
Figure legends

Figure 1: Dynein can bind to dynactin and a cargo adapter such as HOOK3 to form a processive complex (A). Their interaction is facilitated by the coiled-coil in HOOK3. A second dynein can be part of the complex through interactions with the CAPZ $\beta$ subunit of dynactin. Knock-down of CAPZ $\beta$ leads to spindle misorientation. NuMA also possesses a long coiled-coil, which is required for spindle orientation. (B).

Figure 2: Planar spindle orientation can be regulated through different mechanisms. Hertwig's rule states that cells divide according to their long axis (A). Cells with Mud or LGN at TCJ divide according to the position of TCJ (B). In the Drosophila embryo cells adjacent to the actomyosin cable divide according to cell shape if their cell shape is highly anisotropic, but spindle orientation is directed towards the cable in moderately anisotropic cells (C).

Figure 3: In homeostasis, no apparent global direction of cell division can be observed (A). If a stretch is applied to the tissue, the cells become elongated and divide according to their long cell axis (B). Stretched Xenopus animal caps have enriched LGN at TCJ. The anisotropy of the cell as given by TCJ is the best predictor of local stress and cell division orientation (C). Cell divisions in MCDK layers do no follow cell shape, but divide along the direction of stretch by accumulation of LGN on junctions perpendicular to the axis of stretch (D).

Figure 4: Multiple cellular processes have been linked to OCD. In Zebrafish, OCD contribute to the formation of multiple tissue layers in the pre-EVL stage (A). The spreading motion of epiboly generates tissue tension which can be mitigated by OCD along the axis of stretch (B). Similarly, OCD can relieve external stress imposed on MCDK layers (C). In Drosophila, spindle misorientation causes phenotypes in the plane (D) and along the $A B$ axis of epithelia (E). Spindle misorientation in mud mutants leads to smaller wings $(F)$. Furthermore, the loss of $A B$ spindle orientation results in multiple cellular phenotypes. Firstly, a fraction of cells is able to reintegrate into the plane of the tissue. Alternatively, they can be lost through delamination after which the cell can be removed through apoptosis (G). When apoptosis is blocked, these cells can survive and form tumor like masses (G). 
Figure 1

A

Dynein/Dynactin/HOOK3

HOOK3 Dynactin

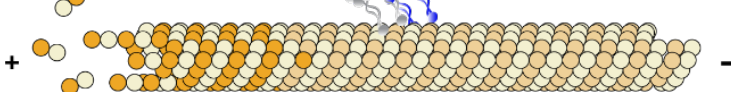

B

Dynein/Dynactin/NuMA?

NuMA?

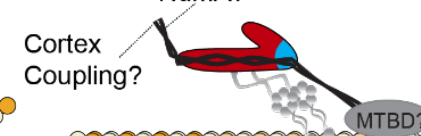

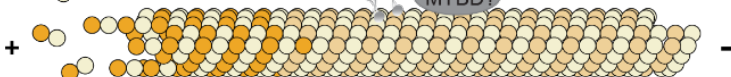

○ ocalsususs

Figure 2

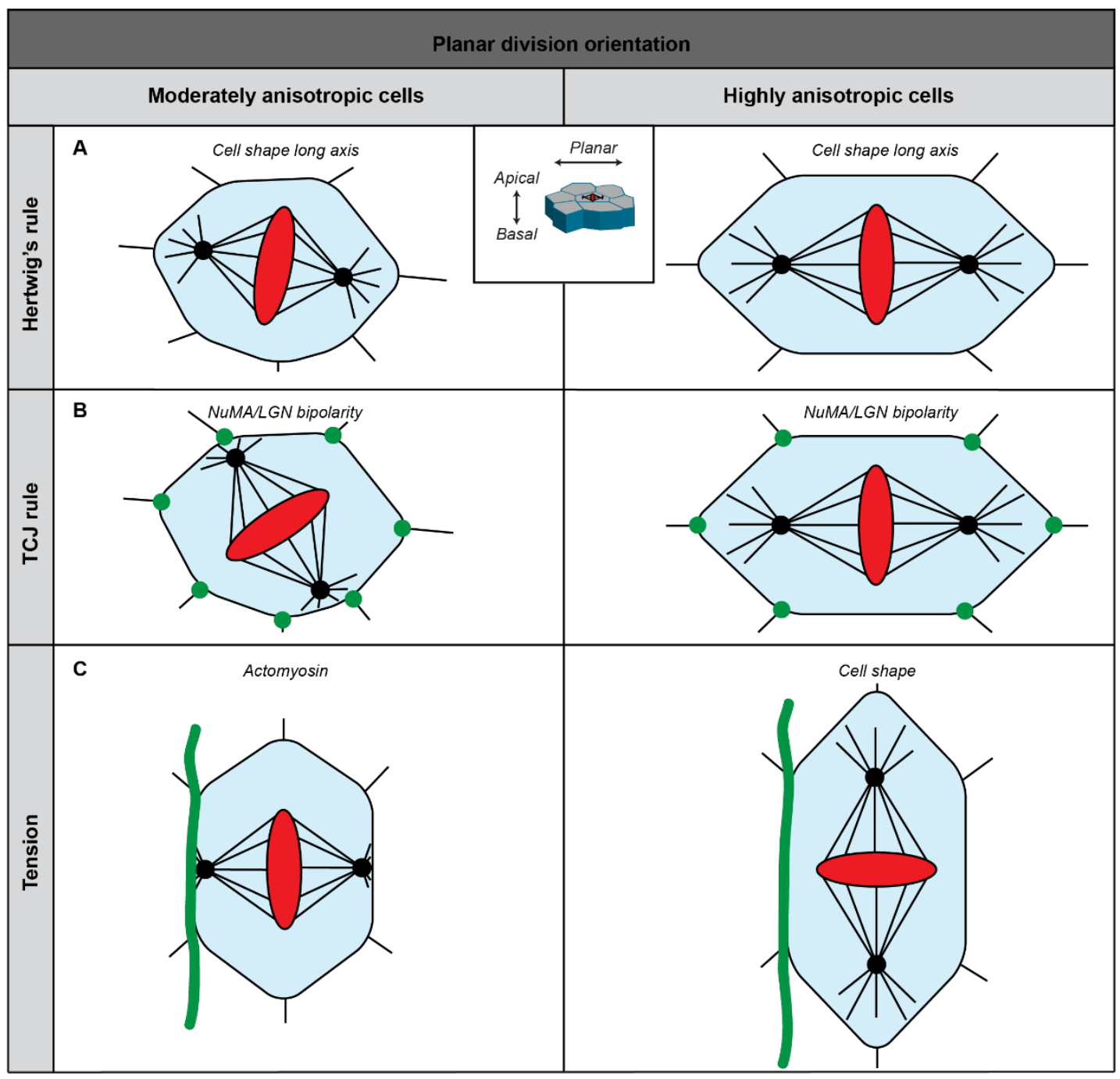


Figure 3

A

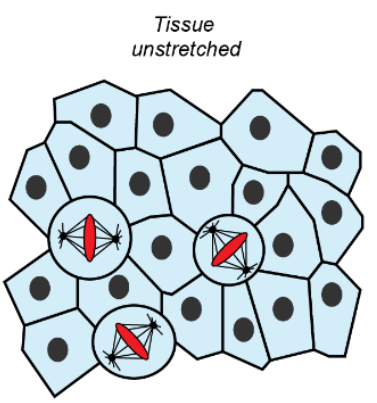

C

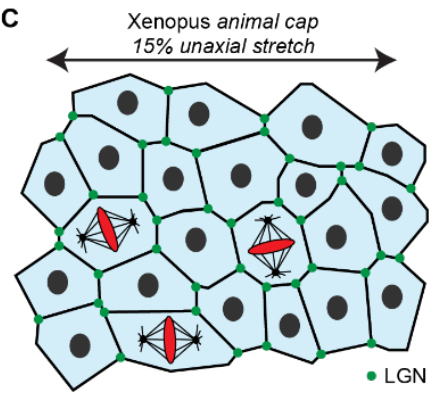

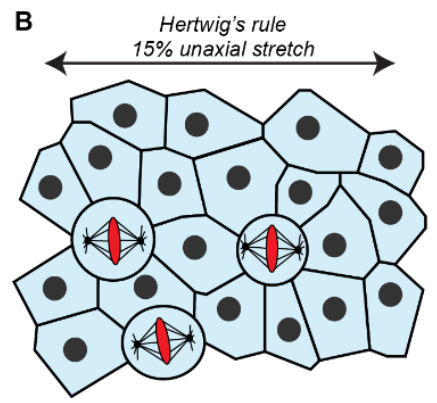

D Madin-Darby Canine Kidney cells

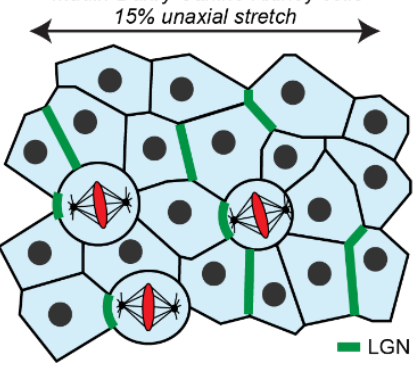

Figure 4

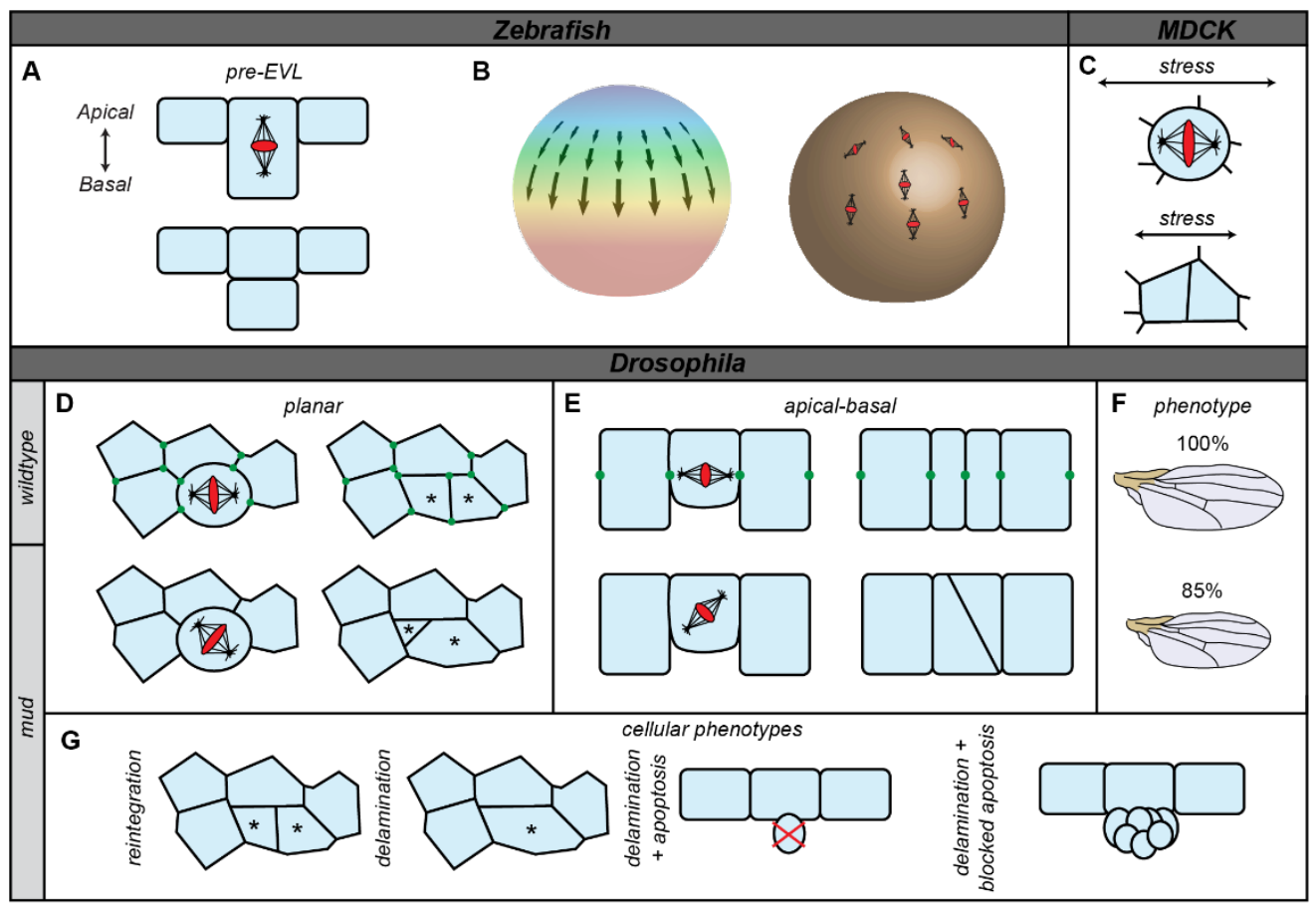


1. Lecuit T, Le Goff L: Orchestrating size and shape during morphogenesis. Nature 2007, 450:189-192.

2. Guirao B, Rigaud SU, Bosveld F, Bailles A, López-Gay J, Ishihara S, Sugimura K, Graner F, Bellaïche Y: Unified quantitative characterization of epithelial tissue development. Elife 2015, 4:1-52.

3. Di Pietro F, Echard A, Morin X: Regulation of mitotic spindle orientation: an integrated view. EMBO Rep 2016, 17:1106-1130.

4. Heisenberg C-P, Bellaïche Y: Forces in Tissue Morphogenesis and Patterning. Cell 2013, 153:948-962.

5. Xiong F, Ma W, Hiscock TW, Mosaliganti KR, Tentner AR, Brakke KA, Rannou N, Gelas A, Souhait L, Swinburne IA, et al.: Interplay of Cell Shape and Division Orientation Promotes Robust Morphogenesis of Developing Epithelia. Cell 2014, 159:415-427.

6. Morin X, Bellaïche Y: Mitotic Spindle Orientation in Asymmetric and Symmetric Cell Divisions during Animal Development. Dev Cell 2011, 21:102-119.

7. Bosveld F, Markova O, Guirao B, Martin C, Wang Z, Pierre A, Balakireva M, Gaugue I, Ainslie A, Christophorou N, et al.: Epithelial tricellular junctions act as interphase cell shape sensors to orient mitosis. Nature 2016, 530:495-498.

8. Grill SW, Gönczy P, Stelzer EHK, Hyman AA: Polarity controls forces governing asymmetric spindle positioning in the Caenorhabditis elegans embryo. Nature 2001, 409:630-633.

9. Gong $\mathrm{Y}$, Mo C, Fraser SE: Planar cell polarity signalling controls cell division orientation during zebrafish gastrulation. Nature 2004, 430:689-693.

10. Mao Y, Tournier AL, Bates PA, Gale JE, Tapon N, Thompson BJ: Planar polarization of the atypical myosin Dachs orients cell divisions in Drosophila. Genes Dev 2011, 25:131-136.

11. Hertwig O: Das Problem der Befruchtung und der Isotropie des Eies, eine Theory der Vererbung. Jenaische Zeitschrift für Naturwiss 1884,

12. Minc N, Burgess D, Chang F: Influence of cell geometry on division-plane positioning. Cell 2011, 144:414-26.

13. Scarpa E, Finet C, Blanchard GB, Sanson B: Actomyosin-Driven Tension at Compartmental Boundaries Orients Cell Division Independently of Cell Geometry In Vivo. Dev Cell 2018, 47:727-740.e6.

${ }^{* *}$ Shows that local tension can orient the mitotic spindle in cells with a mildly anisotropic cell shape in the Drosophila embryo.

14. Finegan TM, Na D, Cammarota C, Skeeters A V, Nádasi TJ, Dawney NS, Fletcher AG, Oakes PW, Bergstralh DT: Tissue tension and not interphase cell shape determines cell division orientation in the Drosophila follicular epithelium. EMBO J 2019, 38:e100072.

* Shows that tissue tension can orient the mitotic spindle in the Drosophila follicular epithelium independently of cell shape.

15. Kiyomitsu T, Cheeseman IM: Cortical Dynein and Asymmetric Membrane Elongation Coordinately Position the Spindle in Anaphase. Cell 2013, 154:391402. 
16. Kotak S, Busso C, Gönczy P: Cortical dynein is critical for proper spindle positioning in human cells. J Cell Biol 2012, 199:97-110.

17. Okumura M, Natsume T, Kanemaki MT, Kiyomitsu T: Dynein-Dynactin-NuMA clusters generate cortical spindle-pulling forces as a multi-arm ensemble. Elife 2018, 7.

** Optogenetically localizes proteins to the cortex in mammalian cells and found that NuMA but not dynein recruitment to the cortex is sufficient to orient the mitotic spindle.

18. Nguyen-Ngoc T, Afshar K, Gönczy P: Coupling of cortical dynein and Ga proteins mediates spindle positioning in Caenorhabditis elegans. Nat Cell Biol 2007, 9:1294-1302.

19. Pirovano L, Culurgioni S, Carminati M, Alfieri A, Monzani S, Cecatiello V, Gaddoni C, Rizzelli F, Foadi J, Pasqualato S, et al.: Hexameric NuMA:LGN structures promote multivalent interactions required for planar epithelial divisions. Nat Commun 2019, 10:2208.

20. Seldin L, Muroyama A, Lechler T: NuMA-microtubule interactions are critical for spindle orientation and the morphogenesis of diverse epidermal structures. Elife 2016, 5.

21. Gallini S, Carminati M, De Mattia F, Pirovano L, Martini E, Oldani A, Asteriti IA, Guarguaglini G, Mapelli M: NuMA Phosphorylation by Aurora-A Orchestrates Spindle Orientation. Curr Biol 2016, 26:458-69.

22. Fielmich L-E, Schmidt R, Dickinson DJ, Goldstein B, Akhmanova A, van den Heuvel S: Optogenetic dissection of mitotic spindle positioning in vivo. Elife 2018, 7.

.* Optogenetically localizes proteins to the cortex in C. elegans and found that NuMA but not dynein recruitment to the cortex is sufficient to orient the mitotic spindle.

23. Couwenbergs C, Labbé J-C, Goulding M, Marty T, Bowerman B, Gotta M: Heterotrimeric $\mathbf{G}$ protein signaling functions with dynein to promote spindle positioning in C. elegans. J Cell Biol 2007, 179:15-22.

24. Siller $\mathrm{KH}$, Doe $\mathrm{CQ}$ : Lis1/dynactin regulates metaphase spindle orientation in Drosophila neuroblasts. Dev Biol 2008, 319:1-9.

25. Yingling J, Youn YH, Darling D, Toyo-Oka K, Pramparo T, Hirotsune S, WynshawBoris A: Neuroepithelial stem cell proliferation requires LIS1 for precise spindle orientation and symmetric division. Cell 2008, 132:474-86.

26. Moon HM, Youn YH, Pemble H, Yingling J, Wittmann T, Wynshaw-Boris A: LIS1 controls mitosis and mitotic spindle organization via the LIS1-NDEL1-dynein complex. Hum Mol Genet 2014, 23:449-66.

27. di Pietro F, Valon L, Li Y, Goïame R, Genovesio A, Morin X: An RNAi Screen in a Novel Model of Oriented Divisions Identifies the Actin-Capping Protein $Z \boldsymbol{\beta}$ as an Essential Regulator of Spindle Orientation. Curr Biol 2017, 27:2452-2464.e8.

28. Urnavicius L, Lau CK, Elshenawy MM, Morales-Rios E, Motz C, Yildiz A, Carter AP: Cryo-EM shows how dynactin recruits two dyneins for faster movement. Nature 2018, 554:202-206.

.${ }^{* *}$ Shows structures of dynein/dynactin/cargo adapter complexes which contain one or two dynein molecules. Complexes with two bound dyneins move at higher speed and generate more force. 
29. McKenney RJ, Huynh W, Tanenbaum ME, Bhabha G, Vale RD: Activation of cytoplasmic dynein motility by dynactin-cargo adapter complexes. Science (80) $2014,345: 337-341$.

30. Olenick MA, Holzbaur ELF: Dynein activators and adaptors at a glance. J Cell Sci 2019, 132:jcs227132.

31. Kiyomitsu T: The cortical force-generating machinery: how cortical spindlepulling forces are generated. Curr Opin Cell Biol 2019, 60:1-8.

32. Kwon M, Bagonis M, Danuser G, Pellman D: Direct Microtubule-Binding by Myosin-10 Orients Centrosomes toward Retraction Fibers and Subcortical Actin Clouds. Dev Cell 2015, 34:323-337.

33. Bowman SK, Neumüller RA, Novatchkova M, Du Q, Knoblich JA: The Drosophila NuMA Homolog Mud Regulates Spindle Orientation in Asymmetric Cell Division. Dev Cell 2006, 10:731-742.

34. Pflüger E: Ueber den Einfluss der Schwerkraft auf die Theilung der Zellen. Arch für die gesamte Physiol 1883,

35. Driesch H: Entwicklungsmechanische Studien. I. Der Werth der beiden Furchungszellen der Echinodermenentwicklung. Zeitschrift für Wissenschaftliche Zool 1892, 53.

36. Fink J, Carpi N, Betz T, Bétard A, Chebah M, Azioune A, Bornens M, Sykes C, Fetler L, Cuvelier D, et al.: External forces control mitotic spindle positioning. Nat Cell Biol 2011, 13:771-778.

37. Théry $M$, Jiménez-Dalmaroni $A$, Racine $V$, Bornens $M$, Jülicher $F$ : Experimental and theoretical study of mitotic spindle orientation. Nature 2007, 447:493-496.

38. Théry M, Racine V, Pépin A, Piel M, Chen Y, Sibarita J-B, Bornens M: The extracellular matrix guides the orientation of the cell division axis. Nat Cell Biol 2005, 7:947-53.

39. Machicoane M, de Frutos CA, Fink J, Rocancourt M, Lombardi Y, Garel S, Piel M, Echard A: SLK-dependent activation of ERMs controls LGN-NuMA localization and spindle orientation. J Cell Biol 2014, 205:791-799.

40. Chanet S, Sharan R, Khan Z, Martin AC: Myosin 2-Induced Mitotic Rounding Enables Columnar Epithelial Cells to Interpret Cortical Spindle Positioning Cues. Curr Biol 2017, 27:3350-3358.e3.

41. Nakajima Y, Meyer EJ, Kroesen A, McKinney SA, Gibson MC: Epithelial junctions maintain tissue architecture by directing planar spindle orientation. Nature 2013 , 500:359-362.

42. Gloerich M, Bianchini JM, Siemers KA, Cohen DJ, Nelson WJ: Cell division orientation is coupled to cell-cell adhesion by the E-cadherin/LGN complex. Nat Commun 2017, 8.

43. McKinley KL, Stuurman N, Royer LA, Schartner C, Castillo-Azofeifa D, Delling M, Klein OD, Vale RD: Cellular aspect ratio and cell division mechanics underlie the patterning of cell progeny in diverse mammalian epithelia. Elife 2018, 7.

44. Box K, Joyce BW, Devenport D: Epithelial geometry regulates spindle orientation and progenitor fate during formation of the mammalian epidermis. Elife 2019, 8.

45. Tamada M, Zallen JA: Square Cell Packing in the Drosophila Embryo through Spatiotemporally Regulated EGF Receptor Signaling. Dev Cell 2015, 
doi:10.1016/j.devcel.2015.09.015.

46. Mao Y, Tournier AL, Hoppe A, Kester L, Thompson BJ, Tapon N: Differential proliferation rates generate patterns of mechanical tension that orient tissue growth. EMBO J 2013, 32:2790-2803.

47. da Silva SM, Vincent J-P: Oriented cell divisions in the extending germband of Drosophila. Development 2007, 134:3049-3054.

48. Hart KC, Tan J, Siemers KA, Sim JY, Pruitt BL, Nelson WJ, Gloerich M: E-cadherin and LGN align epithelial cell divisions with tissue tension independently of cell shape. Proc Natl Acad Sci 2017, 114:E5845-E5853.

** Shows that stretching of MCDK cell layers leads to LGN accumulations on perpendicular bicellular junctions which orients the mitotic spindle with the axis of stretch.

49. Legoff L, Rouault $H$, Lecuit $T$ : A global pattern of mechanical stress polarizes cell divisions and cell shape in the growing Drosophila wing disc. Development 2013, 140:4051-9.

50. Nestor-Bergmann A, Stooke-Vaughan GA, Goddard GK, Starborg T, Jensen OE, Woolner S: Decoupling the Roles of Cell Shape and Mechanical Stress in Orienting and Cueing Epithelial Mitosis. Cell Rep 2019, 26:2088-2100.e4.

** Shows that upon stretch of a multilayer epithelium, spindle orientation does not respond to global tissue stress but is best predicted by the distribution of TCJ enriched in LGN.

51. Stewart MP, Helenius J, Toyoda Y, Ramanathan SP, Muller DJ, Hyman AA: Hydrostatic pressure and the actomyosin cortex drive mitotic cell rounding. Nature 2011, 469:226-231.

52. Sorce B, Escobedo C, Toyoda Y, Stewart MP, Cattin CJ, Newton R, Banerjee I, Stettler A, Roska B, Eaton S, et al.: Mitotic cells contract actomyosin cortex and generate pressure to round against or escape epithelial confinement. Nat Commun 2015, 6:8872.

53. Ramanathan SP, Helenius J, Stewart MP, Cattin CJ, Hyman AA, Muller DJ: Cdk1dependent mitotic enrichment of cortical myosin II promotes cell rounding against confinement. Nat Cell Biol 2015, 17:148-159.

54. Campinho P, Behrndt M, Ranft J, Risler T, Minc N, Heisenberg C-P: Tensionoriented cell divisions limit anisotropic tissue tension in epithelial spreading during zebrafish epiboly. Nat Cell Biol 2013, 15:1405-1414.

55. Wyatt TPJ, Harris AR, Lam M, Cheng Q, Bellis J, Dimitracopoulos A, Kabla AJ, Charras GT, Baum B: Emergence of homeostatic epithelial packing and stress dissipation through divisions oriented along the long cell axis. Proc Natl Acad Sci 2015, 112:5726-5731.

56. Nestor-Bergmann A, Goddard G, Woolner S, Jensen OE: Relating cell shape and mechanical stress in a spatially disordered epithelium using a vertex-based model. Math Med Biol A J IMA 2018, 35:i1-i27.

57. Morin $X$, Jaouen $F$, Durbec $P$ : Control of planar divisions by the G-protein regulator LGN maintains progenitors in the chick neuroepithelium. Nat Neurosci 2007, 10:1440-1448.

58. Ségalen M, Johnston CA, Martin CA, Dumortier JG, Prehoda KE, David NB, Doe CQ, 
Bellaïche Y: The Fz-Dsh Planar Cell Polarity Pathway Induces Oriented Cell Division via Mud/NuMA in Drosophila and Zebrafish. Dev Cell 2010, 19:740-752.

59. Castanon I, Abrami L, Holtzer L, Heisenberg CP, van der Goot FG, González-Gaitán $\mathrm{M}$ : Anthrax toxin receptor $2 \mathrm{a}$ controls mitotic spindle positioning. Nat Cell Biol 2013, 15:28-39.

60. Quesada-Hernández E, Caneparo L, Schneider S, Winkler S, Liebling M, Fraser SE, Heisenberg C-P: Stereotypical Cell Division Orientation Controls Neural Rod Midline Formation in Zebrafish. Curr Biol 2010, 20:1966-1972.

61. Zhou Z, Alégot H, Irvine KD: Oriented Cell Divisions Are Not Required for Drosophila Wing Shape. Curr Biol 2019, 29:856-864.e3.

** Shows that without OCD Drosophila wings are $15 \%$ smaller, look like wildtype and proposes that defects in OCD are compensated by cell rearrangements.

62. Kulukian A, Fuchs E: Spindle orientation and epidermal morphogenesis. Philos Trans R Soc Lond B Biol Sci 2013, 368:20130016.

63. Bergstralh DT, Lovegrove HE, St. Johnston D: Lateral adhesion drives reintegration of misplaced cells into epithelial monolayers. Nat Cell Biol 2015, 17:1497-1503. 